\title{
MOLECULES IN ASTROPHYSICS HALF A CENTURY AGO
}

\author{
H.C. VAN DE HULST \\ Sterrewacht Leiden, P.O. Box 9513 \\ 2300 RA Leiden, The Netherlands
}

I feel a bit embarrassed to be talking about things which happened before most of you were born. But this is what Ewine asked me to do.

To add an element of sport, I suggest that you try a mental game, which often gives amusing results. Many young authors start their professional papers in a rather similar fashion. The introduction of such a paper sounds as follows: "The originally accepted opinion was that things were arranged in such and such a way. But this paper will show that with more complete information we come to quite different conclusions." The game, which any insider in the topic may play, is to guess from the "original ideas" in what year the author of that paper started studying the literature. The story I am about to tell, forms no exception to this rule. It refers to the years of my graduate study, roughly 1940-1945, and fits only that particular period.

My first research job dealing with molecules was to measure the equivalent widths of the lines in the terrestrial $\mathrm{O}_{2}$ oxygen bands in the "Photometric Atlas of the Solar Spectrum". Minnaert had just completed this monumental work after many years of preparation and was eager to see it used. During several years I studied the theory, made the measurements, and derived the curve of growth with the proper run of temperature and pressure in the atmosphere. The results were gratifying, but also inspired me with a deep admiration for the true experts in molecular physics. For a number of questions came sideways to my attention but never received a definite answer: the statistical weights did not match precisely, and there were unknown reactions leading to the formation of $\mathrm{O}_{3}$ or even of $\mathrm{O}_{4}$.

A year later the bulletin board in the Utrecht observatory had the announcement of a prize essay called by the Leiden University. The subject, the growth of interstellar solid particles by gradual condensation of gas, was based on an idea proposed by Lindblad some 5 years earlier. I had passed this announcement several times without paying much attention. Then Minnaert asked me: "Would that not be something for you?" He did 
not know at that moment that soon after that time he would be placed for several years in a hostage camp, reducing his teaching to zero.

The announcement contained one reference, a survey paper by Schoenberg \& Lambrecht (1940), covering lots of topics dealing with interstellar matter in more than 100 pages. In preparation of this talk I have taken this volume again from the library. The part on emission nebulae contains roughly familiar material. The other part, dealing with the solid particles in interstellar space, was at that time fully new to me.

In reading these papers again, two points strike me as peculiar. The first is the absence of any reference to the atomic and molecular absorption lines produced in interstellar space. It seems as if the two authors, in dividing the subject matter, had accidentally left this important issue aside. A review by Swings (1938), which appeared a year earlier, shows that the absorption lines of several atoms and of the molecules $\mathrm{CH}$ and $\mathrm{CN}$ were well established at that time. In my recollection, Swings was also the first to show that the rotational temperature revealed by these lines was $3 \mathrm{~K}$, close to the value predicted by Eddington. But Adams, reviewing the subject a few years later, attributes this finding to McKellar. Anyhow, inferring rotational temperatures from molecular bands had been a routine method, which was applied, e.g., to carbon arcs.

The second peculiar point in Schoenberg's review is the ease by which he assumes (without advancing any particular evidence) that the solid particles must be predominantly iron. Other possibilities, like the ices and frozen gases discussed in the thesis of Greenstein (1937) are mentioned only as a second choice. I now feel that the preference for iron, nickel, and other metals, may have had two grounds. The main reason must have been the knowledge of meteorites, combined with the not yet clearly established separation between interplanetary and interstellar matter. An added reason may be that in the process to match the observed extinction curve to computations by the theory of Mie, metals generally require smaller radii, and therefore shorter and more attractive calculations.

But let me proceed. The university was closed because of the war. The libraries of the institute laboratories (physics, astronomy, and two separate ones for chemistry) were largely deserted, but open. I read at those places about a large variety of things useful and useless. For instance: migration of atoms along a solid surface, condensation nuclei in meteorology, the vapor pressure of droplets of small size, and the accommodation coefficient of gases impinging upon a surface.

I shall mention three conclusions, which I drew at that time and one question that I had to leave open.

My first decision was not to try to develop a theory of the formation of the "first" condensation nuclei of the interstellar particles. The meteo- 
rological literature had scared me away from this topic, because it showed that any number of accidental details, like impurities, could so thoroughly influence the final result that the outcome remained a gamble. However, Kramers, who was with Oort one of the initiators and judges of this prize essay, strongly disagreed. Together with Ter Haar, in whose answer this part of the problem had been emphasized, he performed in the subsequent year a detailed study of the formation of $n$-atomic molecules $(n=2$ to 6 ). In retrospect I have the satisfaction that not much of this study has survived.

My second conclusion led to a more lasting result. It referred to the composition of the particles formed. From the spectroscopy of stars and nebulae, it had become evident in the preceding decennium, that hydrogen played a predominant role in the cosmic abundances. The accommodation tests on cold surfaces made it extremely likely that almost any impinging atom would stick at least initially. Also surface migration of atoms was well established. If this were the full story, it would lead to molecular hydrogen, $\mathrm{H}_{2}$, as the most abundant molecule. But it was also fairly simple to compute from the vapor pressures, which had been accurately measured as functions of temperature, how many molecules evaporate from the surface per unit time and unit area. The conclusion of this calculation was surprisingly simple: At the temperature of these solid particles, which I estimated at more than $3 \mathrm{~K}$, and more likely $10 \mathrm{~K}, \mathrm{H}_{2}$ and $\mathrm{He}$ will largely evaporate from the surface, but any other compound will stick. Because of the surplus of $\mathrm{H}$, mixed with an occasional $\mathrm{O}, \mathrm{C}$, or $\mathrm{N}$ atom, I guessed with my schoolboy knowledge of chemistry that the most probable composition of the particles thus formed would be a mixture of $\mathrm{H}_{2} \mathrm{O}, \mathrm{NH}_{3}$, and $\mathrm{CH}_{4}$, with probably still a certain fraction of $\mathrm{H}_{2}$, that would be retained. When I handed in my essay, this composition seemed a very heretical conclusion, but it also seemed unavoidable to me. Many years later, Greenberg invented for this composition the term "dirty ice". It still is a likely composition of the grain mantles before the processing by ultraviolet radiation or by cosmic rays.

The third conclusion was somewhat disconcerting. Performing the computation of the linear growth of the particle sizes with the then most acceptable gas densities, I found that the particles would grow too fast. During the age of the galaxy they would reach a substantially larger size than their actually observed size. This was a serious discrepancy. Oort and I devoted a continued study to this problem in the following years. In it we speculated about possible causes that would limit or interrupt the growth of the particles. One possibility was the heating by mutual collisions of interstellar clouds, a topic which was very much on Oort's mind at the time. Another possibility seemed to be the periodic entrance into areas of space, where the gas is ionized and the solid particles will evaporate. But I was never 
personally convinced that we had really solved the problem.

The fourth point remained a question. It appeared unavoidable that $\mathrm{H}_{2}$ molecules would continuously be formed by recombination on the surfaces of the solid particles, and then would evaporate. Where did all these molecules go? Spectroscopically, they are virtually invisible and Herzberg saw no obvious chemical processes which will break those molecules down again. The whereabouts of these molecules remained a puzzle. But I remember that in later years, when much attention was paid to the distribution of atomic hydrogen as observed by means of the 21-cm line, Thomas Gold hardly ever missed the opportunity to pronounce in a loud voice from the back of the room: "Let us not forget that there should also be plenty of hydrogen molecules." It took many more years before the CO observations established the existence of molecular clouds. But this topic falls beyond the little piece of history, that I had selected for this talk.

\section{References}

Schoenberg, E., Lambrecht, H. 1940, Interstellare Materie, Ergebnisse d. Exakt. Naturwiss. 19, 1-106

Swings, P. 1938, Les constituants physiques de l'espace interstellaire, Ann. d'Astrophysique 1, 39-59 